\title{
Methotrexate delivery via folate targeted dendrimer-based nanotherapeutic platform
}

\author{
István J. Majoros, ${ }^{*}$ Christopher R. Williams, Andrew Becker and
}

James R. Baker, Jr.

\begin{abstract}
This paper provides a synopsis of the advancements made in advancing a dendrimer-based nanomedicine towards human clinical trials by the Michigan Nanotechnology Institute for Medicine and Biological Sciences. A brief description of the synthesis and characterization of a targeted multifunctional therapeutic will demonstrate the simple yet delicate task of producing novel chemotherapeutic agents. The results obtained from in vitro and in vivo studies not only authenticate the potential of using nanoparticles to target therapeutics but also provide valuable insight towards the future directions of this technology. A fundamental, crossdisciplinary collaboration was necessary to achieve the synthesis and testing of this technology, and was the keystone to establishing this innovative invention. Throughout this paper, we will stress that the unique collaboration that facilitated the evolution of this technology is vital to the success of future developments in nanomedicine. ๑) 2009 John Wiley \& Sons, Inc. WIREs Nanomed Nanobiotechnol 2009 1502-510
\end{abstract}

\section{INTRODUCTION}

$W^{i=n}$ ithin the past few years researchers have begun to realize the dendrimers's potential as supermolecular structures that could facilitate a broad range of biomedical applications. A variety of dendritic molecules have been successfully used for drug and gene delivery, and as protein-receptors, catalysts, and contrast agents. These synthetic nanoscale-molecules, comparable in size to natural body proteins, exhibit many qualities that make them an ideal foundation for medicinal applications. Dendrimers are robust and well-defined macromolecules, offering advantages over similar sized but less stable micelle and liposome molecules. The poly(amidoamine) (PAMAM) dendrimer, the main focus of studies at the University of Michigan, has been of particular interest because of its intrinsic biocompatibility. PAMAM dendrimers are spherical shaped hyper-branched molecules whose success in many applications is particularly encouraging. ${ }^{1,2}$

At Michigan Nanotechnology Institute for Medicine and Biological Sciences (MNIMBS),

\footnotetext{
*Correspondence to: majoros@umich.edu
}

Michigan Nanotechnology Institute for Medicine and Biological Sciences, University of Michigan, Ann Arbor, MI 48109-0533, USA

DOI: $10.1002 /$ wnan.037
PAMAM dendrimer-based drug and imaging agents are being designed as a new generation of therapeutics. Dendrimer-based bioconjugates are innovative smart-drugs which exhibit many desirable effects over conventional therapies. Synthetic nanoscale bioconjugates for cancer therapy are designed to be multifunctional molecules, capable of actively seeking out very specific receptors and killing only the cancer cells. Specific delivery of chemotherapy drugs is made possible as a result of the covalent attachment of both the drug and a targeting moiety to the dendrimer. This controlled delivery allows for smaller doses of drugs to be effective against the cancer and less toxic to healthy cells. Other functional molecules that also have been attached to similar synthetic nanoscale bioconjugates include contrast agents for cellular imaging and apoptosis sensors for immediate feedback of effectiveness. ${ }^{3,4}$

The multifunctional materials described specifically in this review employ a targeting agent, an established chemotherapy drug, and a molecular imaging agent. Folic acid (FA), an essential ingredient in DNA replication, is required in extreme amounts to support the rapid cellular division characteristic of cancer. Many types of cancer, including lung, ovary, colon, and epithelial cancers, over express the high affinity folic acid receptor (FAR). Conjugation of FA to the 
dendrimer provides a means to not only target cancer cells but also to facilitate improved internalization over free chemotherapy drugs. Methotrexate (MTX), a proven chemotherapy drug that has been used for decades, can also easily be conjugated to the dendrimers. By conjugating multiple MTX molecules to each dendrimer the cancer cells are fooled into absorbing high concentrations of the drug. For research purposes, fluorescein isothiocyanate (FITC) was utilized in this multifunctional device as an imaging agent to track the location of the dendrimers. These three functional moieties combined in the proper ratio on a dendrimer create synthetic nanoscale bioconjugates with truly unique functionality. ${ }^{5}$

Successful design and testing of these synthetic nanoscale bioconjugates would not be possible without the cross-disciplinary collaboration that defines MNIMBS. The team of scientists at MNIMBS consists of chemists, physicists, engineers, pharmacists, (bio)informatics specialists, and biologists, all of whom play key roles in capturing a complete understanding of the structure, synthesis, and function of these multifunctional synthetic nanoscale bioconjugates. Every collaborator shares the philosophy that complete understanding of everything that is happening, be it in a test tube, petri dish, or human cancer cell, is the key to producing the most efficient and effective product. Throughout this review, discussion will highlight how cross-disciplinary troubleshooting has expedited the development of the synthetic nanoscale bioconjugates.

\section{DENDRIMER SYNTHESIS}

The synthetic material providing the basis for these bioconjugates is a fifth generation (G5) PAMAM dendrimer. PAMAM dendrimer synthesis (by the divergent method) begins with an ethylenediamine (EDA) initiator core. Repetitive Michael addition of methyl acrylate followed by a condensation (amidation) reaction of the resulting methyl ester with a large excess of EDA produces sequential generations. At higher generations (greater than generation 4), crowding of the branches near the exterior causes the dendrimer to take on a roughly spherical form, and eventually prevents further growth. The result is a highly branched macromolecule with amino groups suitable for drug-linking at its surface (primary), and interior (tertiary). Each reaction sequence theoretically doubles the number of primary amino groups, and molecular weight. $^{6}$ The exact configuration and molecular weight of each dendrimer in every batch cannot be wholly determined, instead, established protocols are followed carefully to ensure that the average dendrimer within a batch is consistent with the desired specifications. Collaboration between chemists and engineers has made dendrimer synthesis quite routine; batches of dendrimers with a specific molecular weight and low polydispersity are easily produced.

\section{SYNTHESIS OF PAMAM DENDRIMER-BASED DEVICES UTILIZING MTX}

The manufacture of the synthetic nanoscale bioconjugates proceeds in the following order, beginning with the G5 PAMAM dendrimer:

1. Partial acetylation of the primary amino groups

2. Reaction with FITC (for tri-functional device for in vitro studies, and control devices)

3. Reaction with FA

4. Glycidolation

5. Reaction with MTX.

Partial acetylation of the dendrimer neutralizes most of the amino groups on its surface. ${ }^{7}$ This is done because while some amino groups will be used for conjugation with the various moieties of the device under construction, not all are necessary for conjugation and leaving them exposed could lead to undesired interactions between the synthetic nanoscale bioconjugates and various molecules of the body. A secondary benefit of the partial acetylation is to increase solubility of the dendrimer, necessary for the reactions with FITC. The stoichiometry of partial acetylation is determined by the dendrimer synthesis so that the average number of acetyl groups for each batch of dendrimer can vary considerably.

According to theoretical chemical structure, ideally there would be exactly 128 primary and 126 tertiary amine groups on a G5 PAMAM dendrimer. In reality, it has been determined by potentiometric titration that the actual average number of primary amines on the dendrimer surface is 110. Acetic anhydride was added to the dendrimer solution in a ratio determined to acetylate an average of 82 primary amines. Acute attention to protocol ensures that each batch is true to the desired product with minimal variance between dendrimers. Once acetylation was complete, acetic acid, a byproduct of the reaction, was removed by membrane filtration and the sample was lyophilized. Purification and characterization of each intermediate is required to determine the proper protocol for each succeeding reaction. 


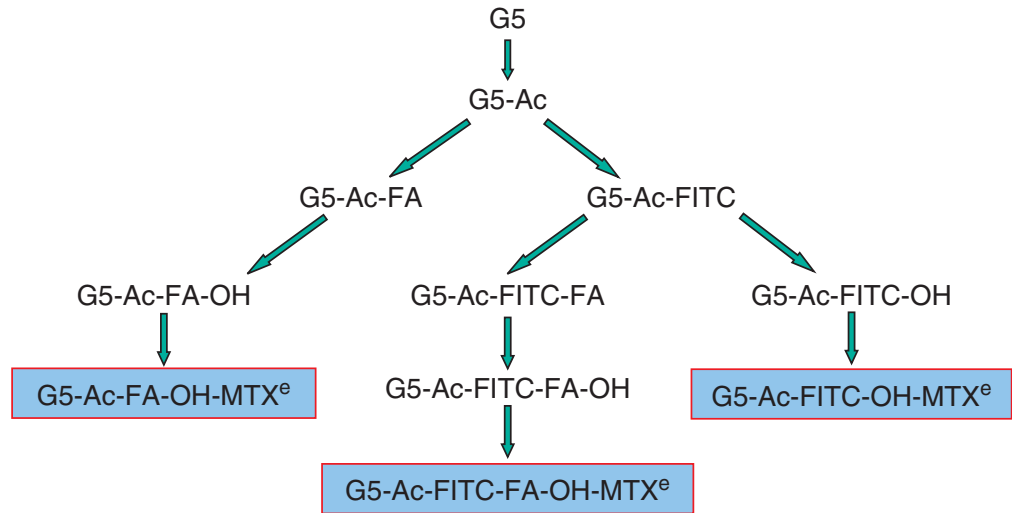

FIG URE 1 | Synthesis pathways leading to three varieties of dendrimer-based, methotrexate-conjugated synthetic nanoscale bioconjugates. The superscript $e$ on MTX highlights its conjugation through an ester bond. From left, G5-Ac-FA-OH-MTXe (without FITC dye) is a clinically applicable device, used for both in vitro and in vivo studies, G5-Ac-FITC-FA-OH-MTXe is an experimental device used to study binding efficacy and cytotoxicity in vitro, and G5-Ac-FITC-OH-MTX ${ }^{e}$ served as a control both for in vitro and in vivo studies.
In the reaction pathway to produce bi-functional devices for in vivo testing, documented in Figure 1, the synthesis begins with the addition of FA. Following the partial acetylation of the dendrimer, FA is attached in a two step process with the $\gamma$-carboxyl group of FA conjugated to the primary amino groups of the dendrimer. This yields the mono-functional device G5Ac-FA. To accomplish this conjugation, an FA-active ester was synthesized through reaction of FA with a 14-fold excess of 1-ethyl-3-(3-dimethylaminopropyl) carbodiimide hydrochloride (EDC) in a mixture of N, N-dimethyl formamide (DMF) and dimethyl sulfoxide (DMSO) at room temperature. This solution was added dropwise to a solution of the partially acetylated G5 dendrimer dissolved in deionized water. Once the desired number of FA has been conjugated to the dendrimer as determined by protocol, the dendrimers will again be purified and lyophilized. By determining the molecular weight of the G5-Ac-FA conjugate through gel permeation chromatography in conjunction with light scattering and refractive index (RI) detection, and comparing that to the molecular weight of G5-Ac, the actual average number of FA conjugated to each dendrimer can be determined. In preparation for the production of bi-functional G5-Ac-FA-MTX, glycidol was added to some of the purified mono-functional device, producing the hydroxyl functionalized G5-Ac-FA-OH necessary for conjugation of MTX via an ester bond.

In order to produce a tri-functional device with an imaging moiety for testing in vitro, the other pathway presented in Figure 1 is followed and the synthesis begins with the addition of FITC. Reaction of FITC with the partially acetylated dendrimer produces mono-functional G5-Ac-FITC. The FITC is covalently attached to the G5 dendrimer by a thiourea bond to a primary amino group. The bi-functional device G5Ac-FITC-FA was formed after the attachment of FA via the previously mentioned process. At this point, a portion of the dendrimers was retained and fully acetylated again as needed for use as a control. The rest of the partially acetylated G5-Ac-FITC and G5-Ac-FITC-FA were glycidolated to form the mono-functional device G5-Ac-FITC-OH, and the bifunctional device G5-Ac-FITC-FA-OH, respectively, converting their remaining primary amino groups to alcohol groups. Again the mono- and bi-functional devices will be purified, lyophilized, and characterized.

MTX was attached via ester bond to the glycidolated dendrimer synthetic nanoscale bioconjugates to form the bi-functional devices G5-Ac-FA-OHMTX $^{e}$ and G5-Ac-FITC-OH-MTX ${ }^{e}$, and the trifunctional device G5-Ac-FITC-FA-OH-MTX ${ }^{e}$. Conjugation of MTX to the dendrimer via ester linkage exhibited preferred cleavage properties compared to amide linkages, which are more stable in acid environments such as endosomes. The MTX is attached by use of EDC chemistry as has been previously described. ${ }^{8}$ The final products were purified by membrane filtration and lyophilized so that they could be easily stored until they were needed.

Although the synthesis of dendrimer-based synthetic nanoscale bioconjugates is performed by chemists, much of the knowledge and understanding that went into the design of these synthetic nanoscale bioconjugates came from contributions from researchers of other disciplines. Utilizing FA as a targeting agent and MTX as a therapeutic was not a random decision, but an educated decision based on knowledge from pharmacologists, biologists, and chemists alike. Cytotoxicity experiments performed by cell biologists lead to the discovery that neutralization of the dendrimer surface charge through acetylation reduces non-specific cytotoxicity. The synthetic nanoscale bioconjugates in this study are specific for cancers that over express the FAR; extension of this technology to synthesize other synthetic nanoscale bioconjugates that can target and treat other types of cancer will require further input from physicians 
and pharmacologists who understand other types of cancers.

\section{CHARACTERIZATION}

Gel permeation chromatography (GPC) with light scattering and refractive index detectors was employed to determine the average number of each conjugated functional group on the tri-functional dendrimer, with an average of 5.8 FITC, 5.7 FA and 5-6 MTX $^{e}$ per dendrimer. These GPC determined numbers are slightly higher than predicted. More precise determination of the conjugate combination was acquired from nuclear magnetic resonance spectroscopy (NMR) and ultraviolet (UV) spectral data. The UV spectra for free FA, MTX and FITC (Figure 2) and the UV spectra of G5-Ac, mono-, bi- and trifunctional dendritic devices (Figure 3) are presented for comparison. The defining peaks for FA appear in Figure 2 at precisely 281 and $349 \mathrm{~nm}$; for MTX at 258, 304 and $374 \mathrm{~nm}$; and for FITC at $493 \mathrm{~nm}$. In
Figure 3, the corresponding distinguishing peaks are dependent on the conjugation of each molecule to the dendrimer. A detailed study of the UV spectroscopic properties of the dendrimer-based bioconjugate is in press. $^{9}$

As the UV spectra of the conjugated molecules are cumulative, comparison of the spectra of free material with the spectra from dendrimerconjugated material was used to establish whether or not the functional molecule(s) in question had actually been attached to the dendrimer. G5-Ac, the carrier dendrimer (Figure 3) demonstrates no characteristic peaks above $300 \mathrm{~nm}$. Conjugation with FITC forms the mono-functional device G5Ac-FITC. The characteristic peak for free FITC $(493 \mathrm{~nm})$ as demonstrated in Figure 2 is shifted to $500 \mathrm{~nm}$ upon conjugation to the carrier dendrimer. Building upon this same principle, the attachment of FA, to form the bi-functional device G5-AcFITC-FA shifts the peak from $349 \mathrm{~nm}$ for free FA to approximately $358 \mathrm{~nm}$ for conjugated FA. The
FIGURE 2 | UV spectra of free FA, MTX, and FITC. (Reprinted with permission from Ref 8. Copyright 2005 ACS).

FIG URE 3 | UV spectra of acetylated G5 PAMAM dendrimer carrier, mono-, bi- and tri-functional devices. (Reprinted with permission from Ref 8. Copyright 2005 ACS).
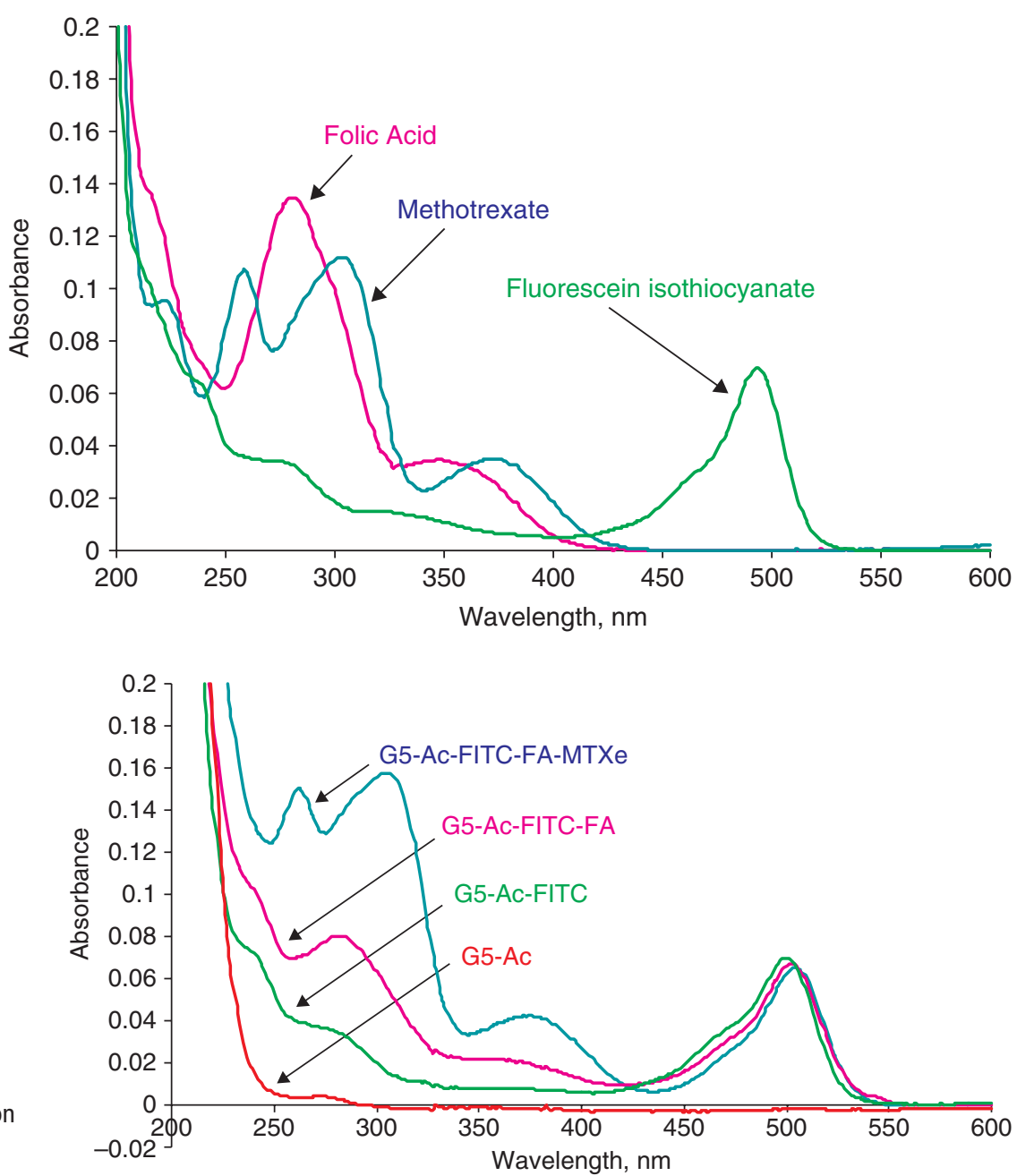
FIG URE 4 | Dose-dependent binding of G5-Ac-FITC-FA-OH-MTXe in KB cells. The cells were maintained under FA-free medium and incubated with different concentrations of the indicated dendrimers for $1 \mathrm{~h}$. The cells were then rinsed and resuspended in PBS and the fluorescence was measured in a flow cytometer. (Reprinted with permission from Ref 13. Copyright 2005 ACS).

location of the other peak, at $281 \mathrm{~nm}$ characteristic of free FA, remains unchanged in the conjugation device while the peak for conjugated FITC is shifted to $502 \mathrm{~nm}$. The tri-functional device G5-AC-FITCFA-MTX ${ }^{e}$ also has slightly shifted peaks. Peaks for conjugate MTX appear at 262 and $304 \mathrm{~nm}$, while the peaks representing a combination of the conjugated MTX and FA converge at $372 \mathrm{~nm}$, and a peak is present at $505 \mathrm{~nm}$ for conjugated FITC. UV characterization required precise purification of the material by filtration to document that there were no free molecules that could confound the analysis. The filtrate did not show absorbance at all wavelengths indicating that the purification removed any free small molecule.

Further characterization of these materials is performed through NMR, high performance liquid

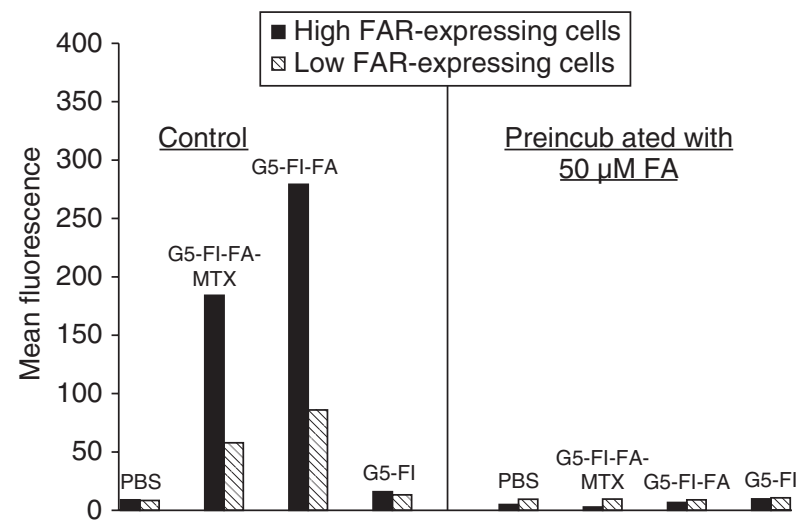

FIG URE 5 | Effect of free folic acid on the uptake of G5-Ac-FITC-FA and G5-Ac-FITC-FA-OH-MTXe in KB cells expressing high and low FAR. $K B$ cells which express high (solid bars) and low (shaded bars) FAR were incubated with $30 \mathrm{nM}$ of the dendrimers for $1 \mathrm{~h}$ at $37^{\circ} \mathrm{C}$, rinsed, and the fluorescence of cells was determined by flow cytometric analysis (left panel). Pre-incubation with $50 \mu \mathrm{M}$ free FA for 30 min prevents cellular binding and uptake (right panel). (Reprinted with permission from Ref 13. Copyright 2005 ACS).

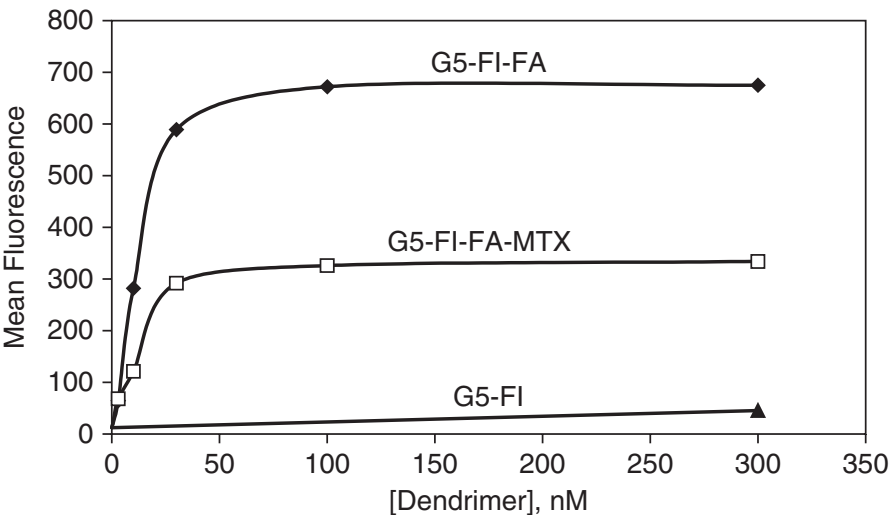

chromatography (HPLC), and capillary electrophoresis $(\mathrm{CE}){ }^{8,10-12} \mathrm{NMR}$ is used to determine the average number of small molecule conjugates, as well as consistency between multiple batches. HPLC and CE serve as quality control techniques to further ensure the purity of the intermediates and final products. The NMR experiments were carried out by physicists while the HPLC and CE were performed by analytical chemists showing the roles different types of scientists play in this work.

\section{BIOLOGICAL FUNCTION STUDIES}

\section{In Vitro Studies}

A preliminary in vitro study of the tri-functional G5Ac-FITC-FA-OH-MTX ${ }^{e}$ was performed to examine cellular uptake and specific cytotoxicity of this material. ${ }^{13,22}$ FITC was not present in the actual planned therapeutic G5-Ac-FA-OH-MTX ${ }^{e}$ but was added to this test material to provide a method of tracing the synthetic nanoscale bioconjugate in vitro. The tri-functional bioconjugate was bound to FA receptor-expressing $\mathrm{KB}$ cells in a dose-dependent and saturable manner. Confocal microscopy was utilized for the analysis of cellular internalization of the conjugates. G5-Ac-FITC-FA-OH-MTX ${ }^{e}$ successfully induced a time- and dose-dependent inhibition of $\mathrm{KB}$ cell growth. While FA targeted devices, G5-Ac-FITCFA-OH-MTX ${ }^{e}$ and G5-Ac-FA-OH-MTX ${ }^{e}$, inhibited cell growth in $\mathrm{KB}$ cells, non-targeted G5-Ac-FITCMTX $^{e}$ (as a control device) neither penetrated the cellular membrane nor induced growth inhibition. These studies illustrate the potential of G5-Ac-FITCFA-OH-MTX ${ }^{e}$ to target and suppress the growth of tumor cells that over express FA-receptors.

\section{CELLULAR UPTAKE}

The two FA targeted materials, G5-Ac-FITC-FAOH-MTX ${ }^{e}$ and G5-Ac-FA-OH-MTX ${ }^{e}$, bound to FA 
over-expressive $\mathrm{KB}$ cells in a dose-dependent manner with $50 \%$ binding at $10-15 \mathrm{nM}$. In comparison, no binding was detected for the control device G5-AcFITC (Figure 4). Analysis of binding kinetics for G5-Ac-FITC-FA-OH-MTX ${ }^{e}(100 \mathrm{nM})$ showed that maximal binding was achieved within $30 \mathrm{~min}$ (data not shown), similar to reports of the binding of free FA.

The effects of free FA on the uptake of the device in $\mathrm{KB}$ cells expressing high and low FAR respectively were tested in order to confirm receptor specificity of the conjugate. ${ }^{14}$ Dendrimer-conjugate binding to low FAR-expressing KB cells was $30 \%$ of that seen with high FAR-expressing cells for both G5-Ac-FITC-FA and G5-Ac-FITC-FA-OH-MTX ${ }^{e}$ (Figure 5, left panel). The uptake of the targeted dendrimers $(30 \mathrm{nM})$ was completely blocked by $50 \mu \mathrm{M}$ FA in both the lowand high-FAR expressing cells (Figure 5).

Confocal microscopy provides further information for the total assessment of the binding and internalization of the conjugates to KB cells. Dendrimerconjugates containing the targeting molecule FA internalized into the cells within $24 \mathrm{~h}$ (Figure 6). As compared to $\mathrm{KB}$ cells treated with the control conjugates (Figure 6(b)), the cells exposed to G5-AcFITC-FA-OH-MTX ${ }^{e}$ (Figure 6(d)), were less adherent and rounded, indicating cytotoxicity induced by the drug-conjugate.

\section{Significance of In Vitro Studies}

Receptor-mediated binding of the conjugate is remarkably efficient in these studies, providing a $\mathrm{Ka}$ of $<10 \mathrm{nM}$. Supporting the results of these experiments are (1) the dose and time-dependent manner in which G5-Ac-FITC-FA-OH-MTX ${ }^{e}$ was bound to FA-expressing cells, (2) the failure of the control dendrimer G5-Ac-FITC to bind to KB cells, (3) the failure of the control dendrimer to associate with KB cells not over-expressing FAR, and (4) the observation that free FA competed with the conjugate for binding (Figures 4 and 5). All of these results lead one to conclude that the conjugate is entering $\mathrm{KB}$ cells through receptor-mediated endocytosis. The concentration-dependent, saturable binding of the conjugate is similar to previous results obtained for the binding of free FA in KB cells. ${ }^{13,15,16}$ Analysis through confocal microscopy demonstrated internalization of the dendrimers and the possibility of utilizing the synthetic nanoscale bioconjugates as an intracellular drug delivery agent.

One of the limiting factors in the use of MTX as a chemotherapeutic drug of choice in our dendrimerconjugate is the development of drug resistance because of the anti-folate properties of MTX. ${ }^{17-20}$ Our results indicate, however, that MTX on the dendrimer surface is carried into the cell through FAR-mediated endocytosis without participation of the reduced folate carrier (RFC) as the conjugate is too large to use this transporter. FAR is known not to be involved in MTX-induced drug resistance; therefore, the drug resistance because of the altered expression of RFC may be overcome in targeted therapy using the conjuage. ${ }^{15}$ Similarly, as the conjugate retention in the cell is independent of polyglutamation, the resistance caused by reduced polyglutamation can be avoided.

The results demonstrate the PAMAM dendrimers are suitable for the targeted delivery of chemotherapeutic molecules capable of killing cancerous cells. Targeted drug delivery may also help to overcome forms of drug resistance to the free drug, and to eliminate non-specific cytotoxicity caused by the free drug interacting with healthy cells.

In vitro studies are the first step in determining the effectiveness of the synthetic nanoscale bioconjugates. As with all new technologies, unexpected problems come up that need to be solved before a product can be deemed usable. Troubleshooting these problems can be a tedious task that requires a deep understanding of the experiments from a number of perspectives. While the cause of the problem may be
FIG URE 6 | Confocal microscopy of KB cells treated with dendrimer-conjugates. $\mathrm{KB}$ cells were incubated with $250 \mathrm{nM}$ of the indicated dendrimers for $24 \mathrm{~h}$ and confocal images were taken. The left and right panels under each treatment represent FITC fluorescence and a differential interference contrast image of the same observation field.

(a) PBS, (b) G5-Ac-FITC, (c) G5-Ac-FITC-FA, and

(d) G5-Ac-FITC-FA-OH-MTXe (Reprinted with permission from Ref 13. Copyright 2005 ACS).
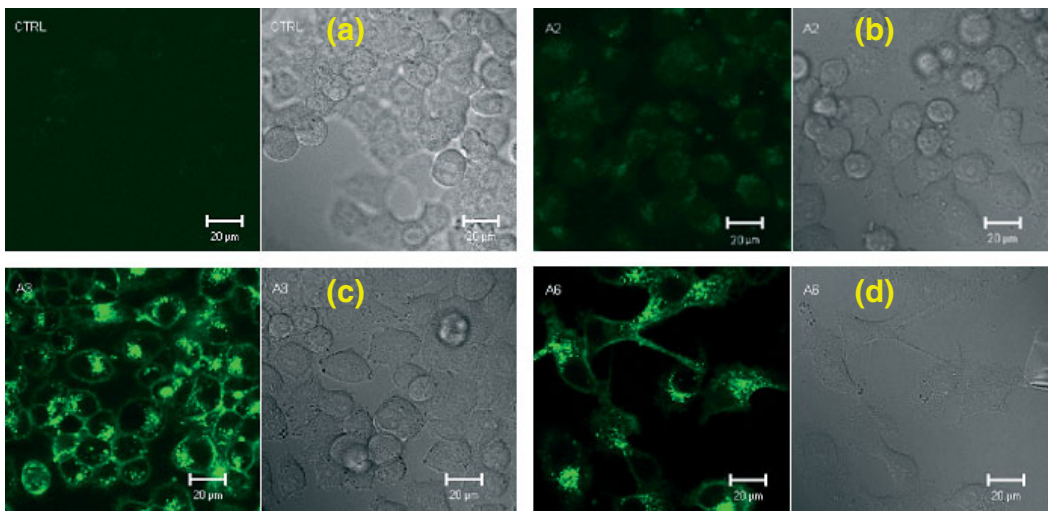
FIG URE 7 | Mice administered either (a) free MTX (15 mg/kg) or (b) G5-Ac-FITC-FA-OH-MTXe (3 mg/kg).

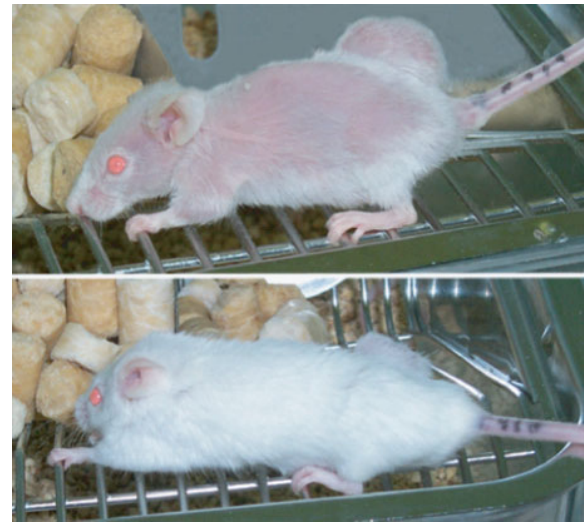

(a) FREE MTX $15 \mathrm{mg} / \mathrm{kg}$ total

(b) SYNTHETIC NANOSCALE BIOCONJUGATES G5-Ac-FITC-FA-OH-MTX $3 \mathrm{mg} / \mathrm{kg}$ total MTX of biological origin, such as FA rescuing of MTXinduced apoptosis, the solution may require the skills of a chemist. In the said case of FA rescue, synthesis of a synthetic nanoscale bioconjugate that contains more MTX than FA, alleviates this problem.

\section{In Vivo Studies}

In vivo mouse trials utilizing saline, free drug or synthetic nanoscale bioconjugates have been performed. The trials served to determine the (1) drug effectiveness utilizing this particular delivery device/method, (2) in vivo targeting capabilities of an FA-conjugated device, and (3) systemic toxicity of the synthetic nanoscale bioconjugates.

Human KB cells over-expressing the high affinity FAR were injected into CB-17 SCID mice at a volume of $5 \times 10^{6} \mathrm{~KB}$ cells in $200 \mu \mathrm{l}$ saline, using animals that had been maintained on a folatedeficient diet for 21 days. Starting on day 4 after tumor cell injection, mice received twice weekly tail vein injection of saline, free drug, or FA targeted MTX synthetic bioconjugates. Injections consisted of doses of equimolar concentrations $(200 \mu \mathrm{l}$ of saline per $20 \mathrm{~g}$ mouse) of free MTX, the experimental synthetic nanoscale bioconjugates (G5-Ac-FITC-FA-OH$\left.\mathrm{MTX}^{e}\right)$, synthetic nanoscale bioconjugates without the imaging molecule FITC (G5-Ac-FA-OH-MTX ${ }^{e}$ ), synthetic nanoscale bioconjugates without the drug MTX (G5-Ac-FITC-FA), synthetic nanoscale bioconjugates without targeting molecule FA (G5-Ac-FITC-OH$\mathrm{MTX}^{e}$ ), or saline as a control. An increased therapeutic index was observed with the targeted conjugate over free drug, allowing better tumor suppression without toxicity.

\section{Significance of In Vivo Studies}

In vivo mouse trials utilizing saline, free drug, and synthetic nanoscale bioconjugates were performed.
The trials served to show (1) drug effectiveness utilizing the synthetic nanoscale bioconjugates delivery method, (2) in vivo targeting capabilities of an FAconjugated device, and (3) systemic toxicity of the synthetic nanoscale bioconjugates.

Administration of free MTX produced intense hair and weight loss, and failed to induce comparable cytotoxicity to the targeted synthetic nanoscale bioconjugates G5-Ac-FITC-FA-OH-MTX ${ }^{e}$ when examined concurrently (Figure $7(\mathrm{a}, \mathrm{b})$ ). The two synthetic nanoscale bioconjugate variants containing $\mathrm{FA}$ and G5-Ac-FITC-FA-OH-MTX ${ }^{e}$ (activity illustrated

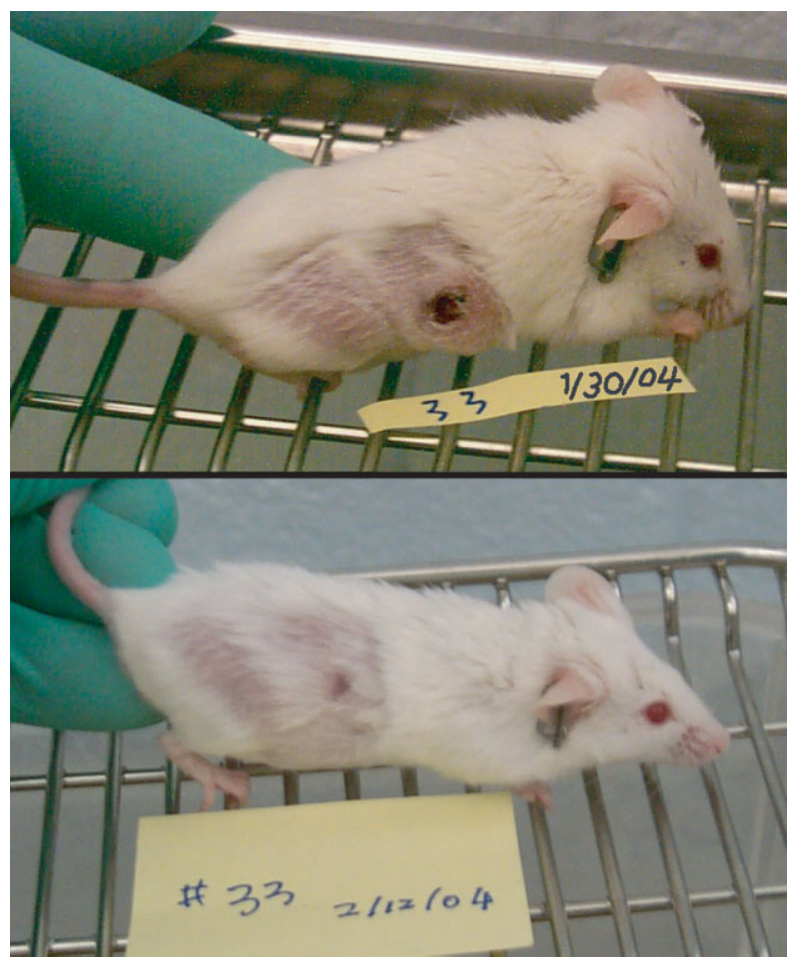

FIG URE 8 | Progress of treatment with G5-Ac-FA-OH-MTXe within a 22-day period. 
in Figure 7) induced a very effective level of cytotoxicity without non-specific toxicity in the animals.

Results of the in vivo mouse trial signify that treatment with the bi- and tri-functional synthetic nanoscale bioconjugates G5-Ac-FA-OH-MTX ${ }^{e}$ and G5-Ac-FITC-FA-OH-MTX ${ }^{e}$, showed statistically significant $(\mathrm{P}<0.01-0.05)$ slowing of tumor growth as compared to treatment with equimolar concentrations of free drug. One significant outcome of this trial occurred on day 39, when a mouse receiving intravenous doses of the synthetic nanoscale bioconjugates G5-Ac-FA-OH-MTX ${ }^{e}$ was completely cured, with the tumor remaining un-palpable for the following 20 days. Figure 8 demonstrates progress of treatment with G5-Ac-FA-OH-MTX ${ }^{e}$ within a 22-day period. Results of this trial confirm the effectiveness of these targeted synthetic nanoscale bioconjugates as chemotherapeutic drug delivery agents. ${ }^{16}$

At 84 days, three out of eight mice were alive in the group receiving G5-Ac-FA-OH-MTX ${ }^{e}$, four out of eight mice were alive in the group receiving G5-AcFITC-FA-OH-MTX ${ }^{e}$, and one mouse out of seven was alive in the group receiving G5-Ac-FITC-FA, the drug free control. Forty-four percent of the mice receiving the drug-containing, targeting, synthetic nanoscale bioconjugates are alive on day 84 , which indicates that these synthetic nanoscale bioconjugates delay tumor growth by at least 30 days on the basis of an end-point volume of $4 \mathrm{~cm}^{3}$. These data have significance for the treatment of human tumors.

Experienced biologists are necessary to perform these types of intricate in vivo studies. Because of the complexity of biological systems, the variability in living animals, and the many factors that can alter the experiment outcome, very detailed and well-thought out experiments must be designed. For both monetary and ethical reasons, experiments must be carried out correctly to avoid unnecessary waste of animals. In vivo studies can be very time consuming and early mistakes are often not be detected until several weeks later. These are reasons why interdisciplinary collaboration is very important for the success of biological nanotechnology. An absolute understanding of all aspects of every experiment will reduce unexpected problems and help to ensure accurate results. Also, a complete understanding of the technology and clear explanation of the results are required to move any medical device through clinical trials and obtain Food and Drug Administration (FDA) approval. Interdisciplinary collaboration leads to the knowledge and skills that are required to ensure the success of any new medical technology. ${ }^{21}$

\section{ACKNOWLEDGEMENT}

Financial support from the National Cancer Institute (No. N01-CM-97065-32) and a SPORE grant from the University of Michigan are gratefully recognized.

\section{REFERENCES}

1. Tomalia DA, Majoros IJ. Dendrimeric supramolecular and supramacromolecular assemblies. In: Ciferri A, ed. Supramolecular Polymers. New York: Marcel Dekker Inc.; 2000, 359-434, Chapter 9.

2. Tomalia DA, Majoros IJ, Dendrimeric supramolecular and supramacromolecular assemblies. Supramol Polym J Macromol Sci C-Polym Rev 2003, C43(3):441-477.

3. Majoros IJ, Thomas TP, Baker JR Jr. Handbook of Theoretical and Co mputational Nanotechnology: Chapter 14. Mol Eng Nanotechnol Eng Drug Deliv 2006, 6:673-717.

4. Patri AK, Majoros IJ, Baker JR Jr. Dendritic polymer macromolecular carriers for drug delivery. Curr Opin Chem Biol 2002, 6:466-471.
5. Baker JR, Quintana A, Piehler L, Banazak-Holl M, Tomalia DA, et al. The synthesis and testing of anticancer therapeutic synthetic nanoscale bioconjugates. Biomed Microdevices 2001, 3:61-69.

6. Majoros IJ, Mehta CB, Baker JR Jr. Mathematical description of dendrimer structure. J Comp Theor Nanosci 2004, 1(2):193-198.

7. Majoros IJ, Keszler B, Woehler S, Bull T, Baker JR Jr. Acetylation of poly(amidoamine) dendrimers. Macromolecules 2003, 36(15):5526-5529.

8. Majoros IJ, Thomas TP, Mehta CB, Baker JR Jr. PAMAM Dendrimer-based Multi-functional engineered synthetic nanoscale bioconjugates for cancer therapy. J Med Chem 2005, 48(19):5892-5899.

9. Majoros IJ, Gopwani SR, Mehta CB, Baker JR Jr. UV-Visibile Spectroscopic Study of Dendritic Multifunctional Synthetic nanoscale bioconjugates, In Press. 
10. Islam M, Majoros IJ, Baker JR Jr. Analysis of PAMAM dendrimer based multifunctional devices. J Chromatogr B Analyt Technol Biomed Life Sci 2005, 822:21-26.

11. Shi X, Majoros IJ, Baker JR Jr. Capillary electrophoresis of Poly(amidoamine) dendrimers: from simple derivatives to complex multi-functional medical synthetic nanoscale bioconjugates. Mol Pharm Rev Artic 2005, 2(4):278-294.

12. Shi X, Majoros IJ, Patri AK, Bi X, Islam MT, et al. Molecular heterogeneity analysis of poly(amidoamine) dendrimer-based mono- and multifunctional synthetic nanoscale bioconjugates by capillary electrophoresis. Anal Res Artic 2005, 2(4):278-294.

13. Thomas TP, Majoros IJ, Kotlyar A, Kukowska-Latallo JF, Bielinksa A, et al. Targeting and inhibition of cell growth by an engineered dendritic synthetic nanoscale bioconjugates. J Med Chem 2005, 48(11):3729-3735.

14. Westerhof GR, Schoranagel JH, Rijnboutt S, Pinedo HM, Janser G. Identification of a reduced folate/methotrexatecarrier in human $\mathrm{Kb}$ cells expressing hogh levels of membrane associated folate binding protein. In: Aying JE, et al. eds. Chemistry and Biology of Pteridines and Folates. New York: Plenum Press; 1993, 771-774.

15. Leamon CP, Reddy JA, Folate-targeted chemotherapy. Adv Drug Deliv Rev 2004, 56:1127-1141.

16. Kukowska-Latallo JF, Candido KA, Cao Z, Nigavekar SS, Majoros IJ, et al. Nanoparticle targeting of anticancer drug improves therapeutic response in animal model of human epithelial cancer. Cancer Res 2005, 65:5317-5324.

17. Sobrero AF, Bertino JR. Endogenousthymidine and hypoxanthine are a surce of error in evaluating methotrexate cytotoxicity by clonogenic assays using undialyzed fetal bovine serum. Int J Cell Cloning 1986, 4:51-62.

18. Roehm NW, Rodgers G, Hatfield S, Glasebrook A. An improved colorimetric assay for cell proliferation and viability utilizing the tetrazolium salt XTT. J Immunol Methods 1991, 142:257-265.

19. White JC, Goldman ID. Methotrexateresistance in an L1210 cell line resulting from increased dihydrofolate reductase, decreased thymidylate synthetase activity, and normal membrane transport. Computer simulations based on network thermodynamics. J Biol Chem $1981,256: 5722-5727$.

20. Kamen BA, Wang M, Streckfuss AJ, Peryea X, Anderson RG. Delivery of folates to the cytoplasm of MA104 cells is mediated by a surface membrane receptor that recycles. J Biol Chem 1988, 263:13602-13609.

21. Majoros IJ, Baker JR Jr. Eds. Dendrimer Based Nanomedicine, Pan Stanford Pb., 2008.

22. Quintana A, Raczka E, Piehler L, Lee I, Myc A, et al. Design and function of dendrimer-based therapeutic synthetic nanoscale bioconjugates targeted to tumor cells through the folate receptor. Pharm Res 2002, 19(9):1310-1316. 